No patients developed an acute kidney injury following angiography.

6 out of $24(25 \%)$ of patients died within 30 days of their IR procedure (figure 3 ).

8 out of $24(33 \%)$ died within 1 year. 3 of these were due to bleeding, 3 due to sepsis and 2 due to malignancies (figure 4).

Conclusions Mesenteric embolisation in patients with significant non-variceal upper GI bleeding has high technical success rates with low rebleeding rates, in a patient population that often is elderly with significant comorbidity.

Approximately one third of patients who undergo interventional radiology procedures for non-variceal upper GI haemorrhage will be dead at 1 year; the majority from non-bleeding related causes.

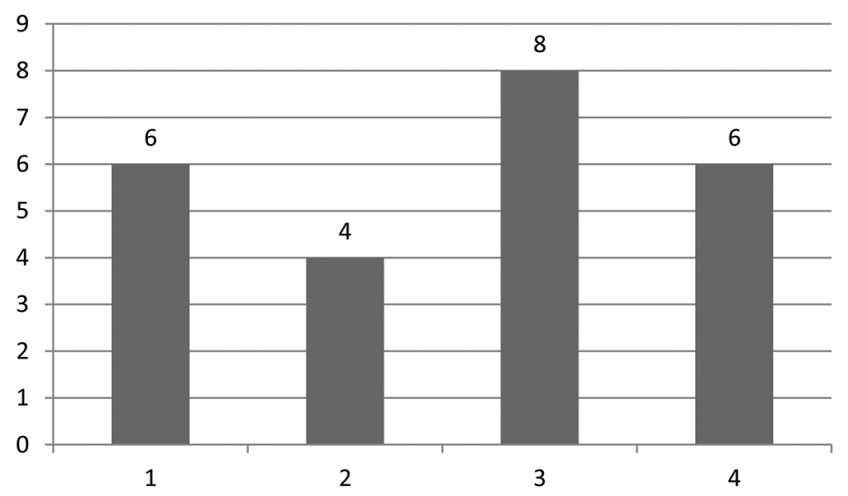

Abstract PTU-019 Figure 1 ASA Grade

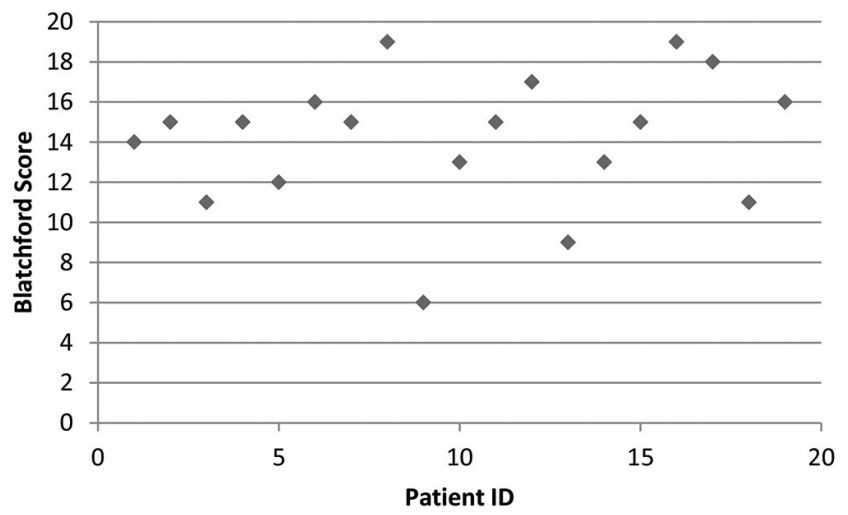

Abstract PTU-019 Figure 2 Glasgow-Blatchford Score

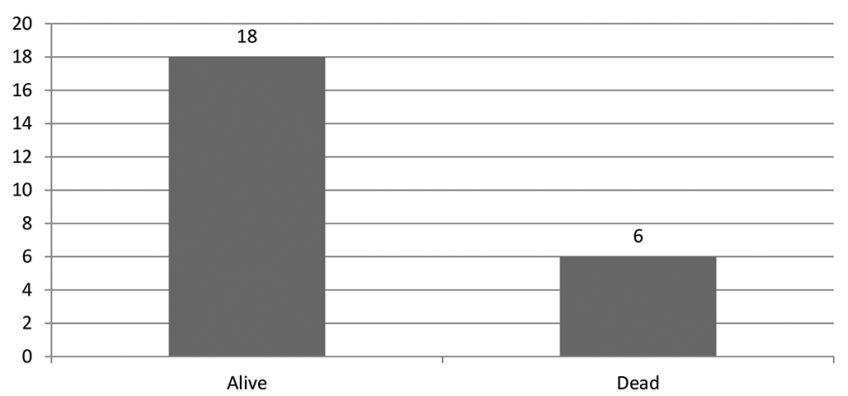

Abstract PTU-019 Figure 3 Mortality at 30 days

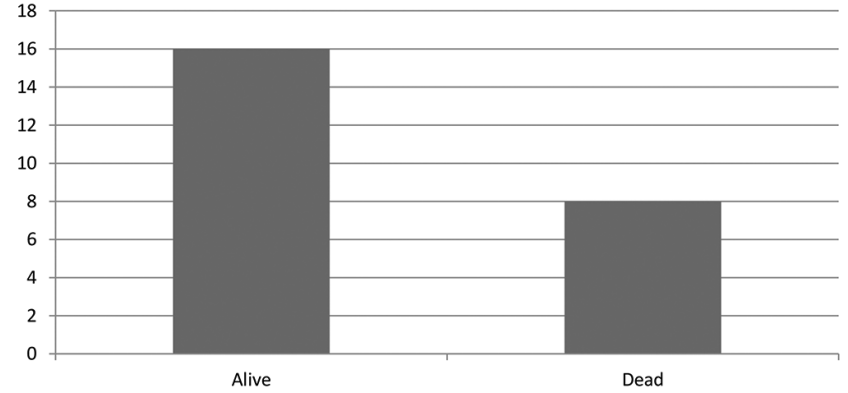

Abstract PTU-019 Figure 4 Mortality at 1 year

\section{PTU-020 INTRODUCTION OF SEMS FOR MALIGNANT DISTAL BILIARY STRICTURES AT A LARGE LONDON DISTRICT GENERAL HOSPITAL}

Emma Dean*, Farhina Sayyed*, Lesley Bain*, Sudeep Tanwar. Whipps Cross Hospital, London, UK

\subsection{6/gutjnl-2018-BSGAbstracts.288}

Introduction Biliary stents are commonly used to treat malignant biliary obstruction. Compared to plastic stents, self-expanding metal stents (SEMS) have a wider diameter and therefore offer enhanced biliary decompression and a longer duration of patency. In addition, biliary decompression with SEMS insertion at ERCP commands a significantly higher level of reimbursement than if a plastic stent is employed. For these reasons, at our hospital since 2015, plastic stents have been abandoned in favour of uncovered or covered SEMS $(60 \mathrm{~mm} \times 10 \mathrm{~mm})$ in patients with unresectable disease or potentially resectable disease respectively. Herein, we report the first 2 years of this change in endoscopic practice at a large DGH in East London.

Methods Patients diagnosed with either pancreatic or biliary tract cancer between April 2015 and April 2017 and who underwent endoscopic biliary stenting were prospectively audited. Retrospective Data collection was performed from electronic systems including Somerset, CRS, EPR and unisoft GI reporting from this prospective cohort.

Results Of 86 patients diagnosed with pancreatic or bile duct cancer, 45 patients (52\%) underwent biliary stenting (37 distal biliary stricture, 9 with a perihilar stricture). Of the 37 with a distal stricture, CBD cannulation rate was 92\%, the remainder required a rendezvous procedure to access the CBD. A SEMS was deployed across the stricture in all cases. A fully covered and uncovered SEMS was deployed in 27 and 12 patient respectively. A $>50 \%$ reduction in bilirubin was identified in $94 \%$ of cases with this effect similar in both covered and uncovered SEMS. Following SEMS insertion 77\% of patients achieved a bilirubin $<50 \mathrm{umol} / \mathrm{L}$. In total, 24 patients required repeat ERCP due to tumour progression with an average of 2.2 interventions per patient. During re-intervention, a new SEMS was deployed within the previous SEMS. 30 mortality post ERCP was 9\%. Mortality at 6 months was 19\%. Distal stent migration was not identified in any patient. Four patients (3 covered and 1 uncovered) suffered cholecystitis due to gallbladder contrast retention after occlusion of the cystic duct orifice. Whereas this was treated with stent removal in patients with covered SEMS, cholecystostomy drainage was required in the patient with uncovered SEMS. 
Conclusions In our unit the introduction of SEMS for the management of distal biliary strictures has resulted in excellent rates of biliary decompression with stent occlusion due to tumour progression managed by SEMS insertion within SEMS. Whereas distal stent migration was not identified in our series, $10 \%$ of patients suffered the gallbladder complications highlighting the need try to avoid SEMS deployment over the cystic duct orifice.

\section{PTU-021 SCREENING FOR COELIAC DISEASE IN ANAEMIA WITH SEROLOGY AND DUODENAL BIOPSIES: SINGLE CENTRED RETROSPECTIVE ANALYSIS}

${ }^{1}$ Arun Sivananthan ${ }^{*},{ }^{2}$ Aruchuna Mohanaruban, ${ }^{3}$ Maryam Bin Desmal, ${ }^{2}$ Simon Peake. ${ }^{1}$ West Middlesex University Hospital, London, UK; ${ }^{2}$ MPerial College NHS Healthcare, London, UK; ${ }^{3}$ Royal College of Surgeons, UK

\subsection{6/gutjnl-2018-BSGAbstracts.289}

Introduction Coeliac disease is an important cause of iron deficiency anaemia with a prevalence of around 1\% and BSG guidance suggests that in suspected individuals a minimum of 4 duodenal biopsies should be taken at endoscopy coupled with coeliac serology testing (tissue tranglutaminase - TTG). The aim of this retrospective study was to determine current practices in coeliac testing for patient undergoing upper GI endoscopy for anaemia at a London NHS Trust.

Methods This was a retrospective study of all upper gastrointestinal endoscopies performed for anaemia over a 3 month period between September and December 2016. The results of TTG serology endoscopy reports and histological findings were analysed and statistical analysis was performed using Microsoft excel.

Results A total of 311 patients underwent upper gastrointestinal endoscopy for anaemia. 2 patients (0.64\%) had biopsy proven coeliac disease (subtotal villous atropy on histology). Both these patients had a positive TTG recorded.

38 patients $(12.2 \%)$ had a TTG recorded prior to endoscopy. 6 patients $(1.89 \%)$ had a positive TTG. 32 patients had a negative TTG. TTG had a sensitivity of $100 \%$, a specificity of $89 \%$, a positive predictive value of $33 \%$ and a negative predictive value of $100 \%$.

\begin{tabular}{lll}
\multicolumn{2}{l}{ Abstract PTU-021 Table $\mathbf{1}$} & \\
\hline & Coeliac & $\begin{array}{l}\text { Not } \\
\text { Coeliac }\end{array}$ \\
\hline TTG & 2 & 4 \\
+ve & 2 & 32 \\
TTG - & 0 & \\
ve & & \\
\hline
\end{tabular}

210 patients $(67.5 \%)$ had duodenal biopsies performed. Of these $178(84.76 \%)$ had 4 or more duodenal biopsies.

Of 21 patients who had a negative TTG before endoscopy $12(57.14 \%)$ had biopsies. None of these patients were found to have coeliac disease.
Conclusions This study demonstrates that the majority of patients receive 4 or more duodenal biopsies at endoscopy as recommended in the guidelines. In addition we have evidence that TTG serology appears a useful negative predictive test which is rarely available prior to endoscopy. Prior testing will help guide the endoscopist and may help avoid costly and unnecessary duodenal biopsies when investigating anaemia. Therefore the uptake of coeliac antibody testing should be encouraged in patients being investigated for anaemia.

\section{PTH-116 BARRETT'S NEOPLASIA DETECTION: SYSTEMATIC OR TARGETED BIOPSY?}

${ }^{1}$ Arun Nachiappan*, 2,3 Timothy Card, ${ }^{4}$ Hemant Gupta, ${ }^{5}$ Saqib Ahmad, ${ }^{3}$ Krish Ragunath, ${ }^{3,6}$ Philip Kaye. ${ }^{1}$ Nottingham University Hospitals NHS Trust, Nottingham, UK; ${ }^{2}$ Division of Epidemiology and Public Health, Medical School, Queen's Medical Centre, University of Nottingham, Nottingham, UK; ${ }^{3}$ NIHR Nottingham Digestive Diseases Biomedical Research Centre, Nottingham University Hospitals NHS Trust, Nottingham, UK; ${ }^{4}$ United Lincolnshire Hospitals NHS Trust, UK; ${ }^{5}$ Sherwood Forest Hospitals NHS Foundation Trust, UK; ${ }^{6}$ Department of Pathology, Nottingham University Hospitals NHS Trust, Nottingham, UK

\subsection{6/gutjnl-2018-BSGAbstracts.290}

Introduction The role of systematic biopsy (i.e. random, fourquadrant biopsy) in Barrett's oesophagus surveillance has come under question given its drawbacks and the emergence of high-resolution endoscopy plus advanced imaging modalities. Our study aims to assess whether neoplastic pathology is typically diagnosed by systematic or targeted biopsy whilst using high-resolution endoscopy.

Methods A retrospective analysis of patients diagnosed with Barrett's oesophagus with dysplasia or neoplasia at a tertiary referral centre from 2008 onwards. Endoscopic and histopathologic data pertaining to the initial endoscopy in which pathology was diagnosed was extracted from the medical records. The most advanced histopathologic abnormality at initial diagnosis and within twelve months were noted. The corresponding endoscopic impression at initial diagnosis was used to group cases per type of biopsy - systematic or targeted. Pearson's chi-squared test of independence was used to analyse the relationship between the type of biopsy and diagnosis in twelve months, indication for endoscopy, endoscopist level and advanced techniques used.

Results Of the 222 patients involved in the study - a higher proportion were diagnosed through systematic biopsy $(72.97 \%)$ than targeted biopsy $(27.03 \%) ; \chi^{2}$ (degrees of free$\operatorname{dom}[\mathrm{df}]=2, \mathrm{n}=222)=31.56, \mathrm{p}<0.001$. 90.91\% of low-grade dysplasia $\left(\chi^{2}[\mathrm{df}=2, \mathrm{n}=88]=4.91, \mathrm{p}=0.086\right), 71.43 \%$ of highgrade dysplasia $\left(\chi^{2}[\mathrm{df}=2, \mathrm{n}=70]=11.58, \mathrm{p}=0.003\right)$ and $50 \%$ of intramucosal adenocarcinoma $\left(\chi^{2} \quad[\mathrm{df}=2, \mathrm{n}=64]=5.18\right.$, $\mathrm{p}=0.075)$ cases were diagnosed by systematic biopsy. Across all grades of clinicians, patients were typically diagnosed through systematic biopsy; $\chi^{2}(\mathrm{df}=3, \mathrm{n}=215)=4.68, \mathrm{p}=0.322$. However, amongst specialist consultant endoscopists $(\mathrm{n}=10)$ the proportion was equal.

Conclusions Our findings strongly emphasise the importance of systematic biopsy in the detection of not only low-grade dysplasia, but also high-grade dysplasia and early invasive carcinoma as part of Barrett's oesophagus surveillance. 